\title{
Effect of the Laser Wave Front in a Laser-Plasma Accelerator
}

\author{
B. Beaurepaire, A. Vernier, M. Bocoum, F. Böhle, A. Jullien, J-P. Rousseau, T. Lefrou, D. Douillet, \\ G. Iaquaniello, R. Lopez-Martens, A. Lifschitz, and J. Faure \\ Laboratoire d'Optique Appliquée, UMR 7639, ENSTA-CNRS-Ecole Polytechnique, \\ 91761 Palaiseau, France
}

(Received 10 December 2014; published 31 July 2015)

\begin{abstract}
A high-repetition rate electron source is generated by tightly focusing $\mathrm{kHz}$, few-mJ laser pulses into an underdense plasma. This high-intensity laser-plasma interaction leads to stable electron beams over several hours but with strikingly complex transverse distributions even for good quality laser focal spots. We find that the electron beam distribution is sensitive to the laser wave front via the laser midfield distribution rather than the laser focal spot itself. We are able to measure the laser wave front around the focus and include it in realistic particle-in-cell simulations demonstrating the role of the laser wave front on the acceleration of electrons. Distortions of the laser wave front cause spatial inhomogeneities in the midfield laser intensity and, consequently, the laser pulse drives an inhomogeneous transverse wakefield whose focusing and defocusing properties affect the electron distribution. These findings explain the experimental results and suggest the possibility of controlling the electron spatial distribution in laser-plasma accelerators by tailoring the laser wave front.
\end{abstract}

DOI: 10.1103/PhysRevX.5.031012

\section{INTRODUCTION}

Laser-plasma accelerators [1,2] currently provide $100 \mathrm{MeV}$ to few $\mathrm{GeV}$ electron beams in short distances $[3,4]$, owing to their very high accelerating gradients $\simeq 100 \mathrm{GV} / \mathrm{m}$. In these experiments, an intense laser pulse drives a large amplitude plasma wave in which plasma electrons can be trapped and accelerated to relativistic energies in millimeter distances. These laser-driven electron sources are also of great interest because the generated electron bunches can be extremely short, with durations reaching down to a few femtoseconds only [5]. In general, laser-plasma accelerators operate using 100-TW, joulelevel laser systems. In this case, the laser power exceeds the critical power for relativistic self-focusing [6,7], $P / P_{c}>1$, and the laser pulses are self-focused over several Rayleigh lengths. Injection and acceleration of electrons occur in the region where the laser is self-focused, corresponding to the region where the plasma wave amplitude is high. In many experiments, the laser wave front is corrected in order to obtain the best laser spot in the focal plane and little attention is given to the laser transverse distribution outside the focal plane.

Recently, several groups have been developing laserplasma accelerators operating at high-repetition rate, using $\mathrm{kHz}$ lasers with energies $<10 \mathrm{~mJ}[8,9]$. Such developments are particularly important for applications as the

Published by the American Physical Society under the terms of the Creative Commons Attribution 3.0 License. Further distribution of this work must maintain attribution to the author(s) and the published article's title, journal citation, and DOI.
Subject Areas: Optics, Plasma Physics

high-repetition rate improves the beam stability and permits data accumulation. Indeed, $\mathrm{kHz}$ electron beams with femtosecond duration and energies in the $\mathrm{MeV}$ range are of great interest for ultrafast electron diffraction [10,11], a powerful technique for investigating structural dynamics in matter with femtosecond resolution. Recent experiments have shown the possibility of generating $100-\mathrm{keV}$ electron bunches at $\mathrm{kHz}$ repetition rate [8]. Electron bunches from a $\mathrm{kHz}$ laser-plasma source were successfully used to obtain clear diffraction patterns on single crystal gold foils [12]. In these high-repetition rate experiments, the laser pulse has to be focused very tightly in order to reach intensities in excess of $10^{18} \mathrm{~W} / \mathrm{cm}^{2}$, as required to drive large amplitude plasma waves. Therefore, the Rayleigh range of the laser beam is extremely short, typically on the order of $z_{R} \simeq 10 \mu \mathrm{m}$. In addition, self-focusing does not occur because the power is not high enough, $P / P_{c}<1$. Consequently, the interaction region extends outside the focal region and one expects that experimental results should also depend critically on the laser distribution in the intermediate field.

In this article, we show results from a laser-plasma accelerator operating at $\mathrm{kHz}$ repetition rate. The interaction region is large compared to the Rayleigh range, $L \gg z_{R}$, so that the laser distribution outside the focal region plays an important role in the physics of electron injection and acceleration. We demonstrate that the spatial distribution of the electron beam originates from the inhomogeneity of the laser distribution outside the focal region, due to an imperfect laser wave front. Particle-in-cell (PIC) simulations show that an inhomogeneous laser pulse drives an inhomogeneous transverse wakefield, which affects the 
electron beam quality through its focusing and defocusing properties. Our work emphasizes the influence of the laser wave front over wake excitation as well as on the electron beam spatial distribution.

\section{EXPERIMENT}

A schematic of the experiment is shown in Fig. 1. The experiment is performed using $1-\mathrm{kHz}, 800-\mathrm{nm}$ laser pulses with $\tau=22$ fs duration at FWHM. The energy on target is in the range $2.5-3 \mathrm{~mJ}$. The laser system provides highcontrast laser pulses and good beam quality through the use of spatial filtering in a hollow core fiber [13]. The laser pulses are focused down to a spot size of $\lesssim 2 \mu \mathrm{m}$ FWHM using a $f \# 1.5,90^{\circ}$ off-axis parabolic mirror, thus leading to a peak intensity of $I=(2.5-3) \times 10^{18} \mathrm{~W} / \mathrm{cm}^{2}$ and a measured Rayleigh range of $\simeq 20 \mu \mathrm{m}$. The gas target consists of a $100-\mu \mathrm{m}$ diameter gas jet operating in continuous flow so as to make $\mathrm{kHz}$ operation possible. The electron beam profile is measured using a $\mathrm{CsI}(\mathrm{Tl})$ phosphor screen imaged onto a 14-bit CCD camera. The electron energy distribution is measured by inserting a magnetic electron spectrometer. Information on laser propagation in the gas jet is obtained by imaging the transmitted laser mode at the exit of the plasma. Finally, the electron density is monitored in situ by transverse interferometry with a $22-\mathrm{fs}$ probe laser pulse. The experiment is run at $\mathrm{kHz}$ repetition rate and all data shown in this paper are averaged over 500-1000 shots.

The data are taken using nitrogen as we are limited by the evacuation of the gas fed into the jet. Gas evacuation is a critical issue in this experiment since we need, on the one hand, a continuous gas flow to make full use of the highrepetition rate of the laser, and, on the other hand, a fairly high electron density $\left(n_{e}>10^{19} \mathrm{~cm}^{-3}\right)$. The required electron density is reached with nitrogen for a backing pressure about 5 times lower than with helium, and considering the inefficiency of the pumping of helium

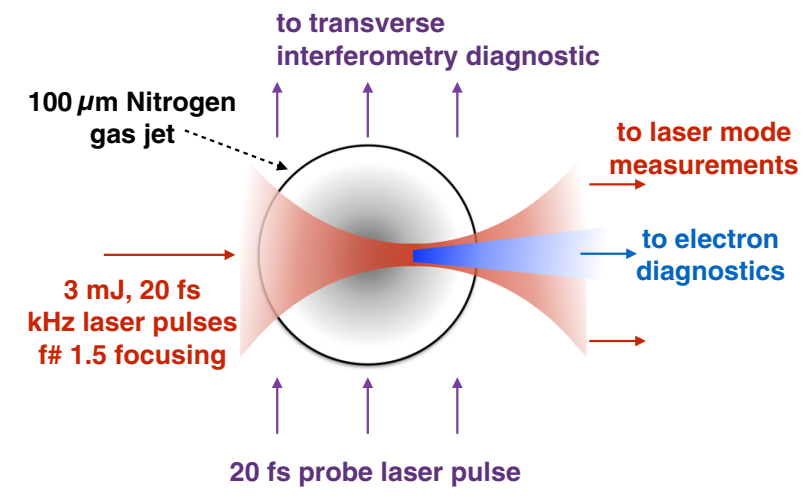

FIG. 1. Top view schematic of the experimental setup. A laser pulse is tightly focused into a gas jet. Strong focusing with a $f \# 1.5$ optics results in a short Rayleigh length compared to the gas jet length of $100 \mu \mathrm{m}$. compared to nitrogen, using the latter allows us to get the most complete data set.

In nitrogen, the first five electrons are ionized at intensities $I \lesssim 10^{16} \mathrm{~W} / \mathrm{cm}^{2}$, so that ionization-induced defocusing $[14,15]$ does not significantly affect the laser pulse propagation for $I \simeq 10^{18} \mathrm{~W} / \mathrm{cm}^{2}$. However, away from focus $\left(L \gg z_{R}\right)$, the laser intensity in the gas jet can decrease down to the $10^{16} \mathrm{~W} / \mathrm{cm}^{2}$ range and ionization might affect propagation. By measuring the transmitted laser mode, we check that the laser pulse does not undergo filamentation and that ionizationinduced defocusing $[14,15]$ does not significantly affect the position and size of the focal spot (see Supplemental Material [16]). Therefore, even in the presence of ionization, the laser pulse reaches intensities in excess of $10^{18} \mathrm{~W} / \mathrm{cm}^{2}$, as required for generating large amplitude plasma waves.

The green curve in Fig. 2(a) represents a typical longitudinal electron density profile when the laser is focused $150 \mu \mathrm{m}$ above the gas jet. Here, the density reaches $n_{e}=3 \times 10^{19} \mathrm{~cm}^{-3}$ and the density gradients are on the order of $50 \mu \mathrm{m}$. The blue curve in Fig. 2(a) represents the measured accelerated charge as a function of the position of the laser focus. We find that the amount of accelerated electrons depends critically on the position of the focal plane, and that a charge as high as $20 \mathrm{fC} / \mathrm{shot}$ (i.e., $20 \mathrm{pC} / \mathrm{s}$ ) can be obtained when the laser is focused in the density down ramp. In Fig. 2(b), the accelerated charge is plotted as a function of $\omega_{p}(z) \tau$, where $\omega_{p}(z)=$ $\sqrt{n_{e}(z) e^{2} / m_{e} \epsilon_{0}}$ is the local plasma frequency along the propagation axis. The green curve represents the plasma wave amplitude $\Delta \phi$ obtained from a 1D nonlinear fluid model of plasma wave excitation [17]. The experimental data peak at $\omega_{p} \tau \approx 2.6$, which is very close to the theoretical estimate of resonant plasma wave excitation; i.e., for a 22 -fs laser pulse, the resonant density is $n_{e}^{\text {res }} \simeq 5 \times 10^{18} \mathrm{~cm}^{-3}$. This last result indicates that electrons are produced preferentially when the resonant condition is satisfied, suggesting electron acceleration by plasma waves. In addition, electrons are produced only when the laser is focused in the density down ramp, pointing toward density gradient injection [18-22], as reported previously in a similar experiment in Ref. [8]. Thus, the experimental evidence tends to show that the excitation of a large amplitude plasma wave in the density gradient results in trapping and subsequent acceleration. This interpretation is confirmed by the simulations shown Sec. III.

Comparable results were obtained using helium instead of nitrogen, excluding ionization injection $[23,24]$ as a possible injection mechanism. Finally, we measure that the accelerated electrons have a large energy distribution extending to about $100 \mathrm{keV}$; see Fig. 2(f). Note that electrons below $50 \mathrm{keV}$ are not detected because our $\mathrm{CsI}(\mathrm{Tl})$ detector has a very small response below $50 \mathrm{keV}$. 
(a)

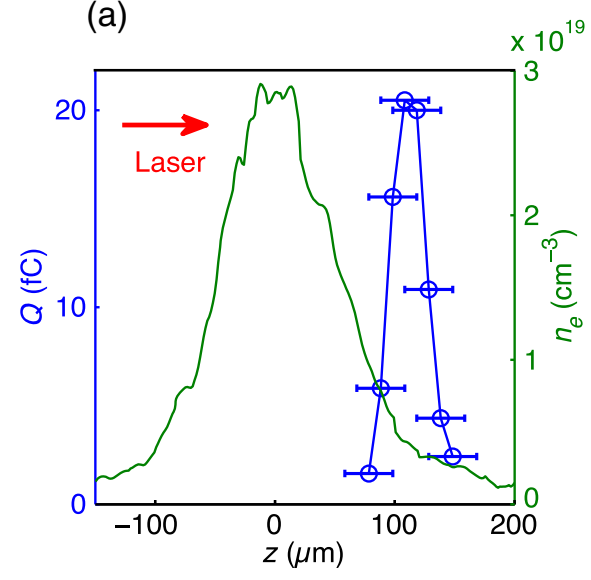

(c)

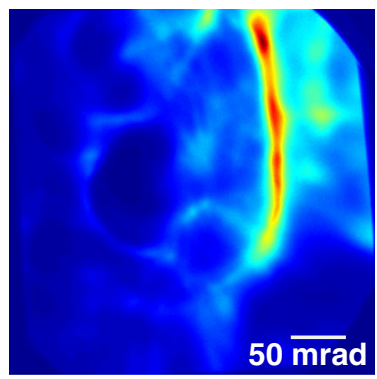

(d) (b)

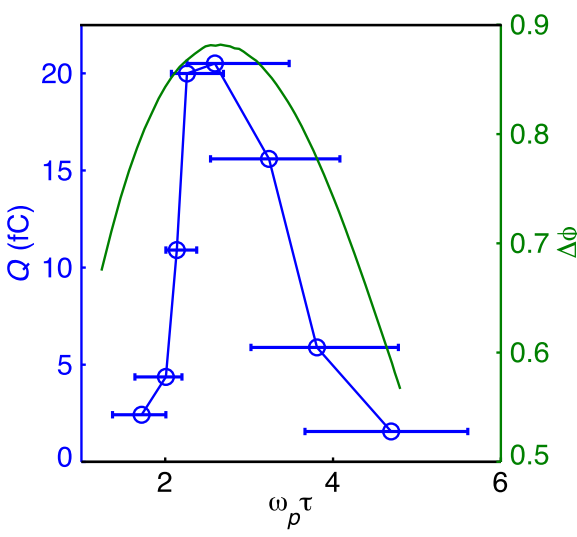

(e)
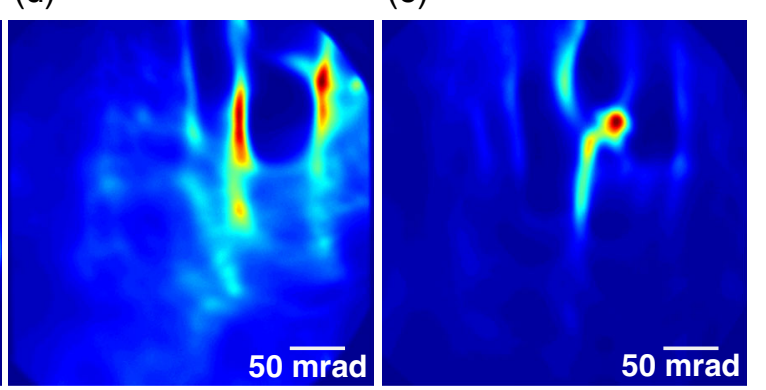

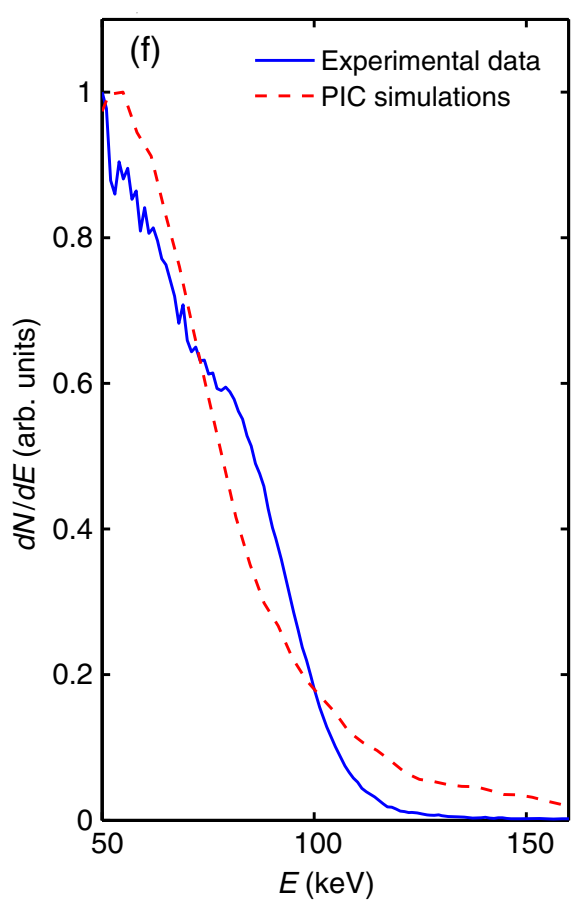

FIG. 2. Experimental results. (a) Green curve, measured longitudinal electron density profile; blue curve, charge or shot of the accelerated electrons for different positions of the laser focus. (b) Beam charge as a function of $\omega_{p}(z) \tau$, where $\omega_{p}(z)$ is estimated using the experimental plasma density at the focal plane. The green curve is the theoretical plasma wave amplitude $\Delta \phi$. Horizontal error bars originate from a $\pm 20 \mu \mathrm{m}$ uncertainty on the position of the focus. (c)-(e) Typical images of electron beam profiles. (f) Electron energy distribution. Blue line, experimental result; red line, distribution from the 3D PIC simulation.

Figures 2(c)-2(e) show typical electron beam transverse distributions obtained during different experimental runs. Each image is obtained by averaging over about 1000 shots. These beams have complex structures that are not random and are remarkably stable over the course of several hours. In these $\mathrm{kHz}$ experiments, the electron beam parameters vary very little for a given set of experimental conditions (see also Ref. [12]): the charge fluctuations are on the order of 7\% rms and the energy and divergence fluctuate at the percent level [25]. The shape of the beam does, however, change from run to run. Depending on the experimental conditions, it can exhibit various complex patterns [see Figs. 2(c) and 2(d)] or a low-divergence beam $<10 \mathrm{mrad}$ and containing $\simeq 20 \mathrm{fC}$, as in Fig. 2(e). Such electron distributions are quite different from those emerging from high-energy laserplasma accelerators. Similar beams were obtained in Ref. [8], but no explanation was given for the observed complex structures.

Experimentally, we notice that the electron beam spatial structures are correlated to the laser distribution in the intermediate field rather than to the laser distribution at focus. Indeed, similar focal spots produce very different electron beams when the midfield laser distribution changes significantly. For instance, Figs. 3(a) and 3(b) show the laser beam at focus for two different runs that result in different electron beams. Both laser beams have a $\sim 2 \mu \mathrm{m}$ diameter FWHM, and the laser intensity is similar. In contrast, the differences between the laser distributions at an intermediate plane $\left[z=2 z_{R}\right.$, Fig. 3(c)] are conspicuous. The strong degradation of the beam quality far from the focal plane indicates the presence of significant wave front distortions, which can vary from run to run.

The wave front is reconstructed numerically from the laser intensity distribution at the two planes shown in Fig. 3 $\left(z=0\right.$ and $\left.z=2 z_{R}=40 \mu \mathrm{m}\right)$ using the Gerchberg-Saxton algorithm [26]. To test the accuracy of the result, the obtained phase is used to reconstruct the intensity distribution before the focal plane (at $z=-2 z_{R}$ ). The extrapolated intensity is in good agreement with the experimentally measured laser distribution in this plane. The wave fronts obtained at the focal plane are shown in Figs. 3(e) and 3(f). They exhibit complex structures, which are quite different for the two runs. These data suggest that the variation of the laser wave front has a direct influence over the electron beam distribution. 

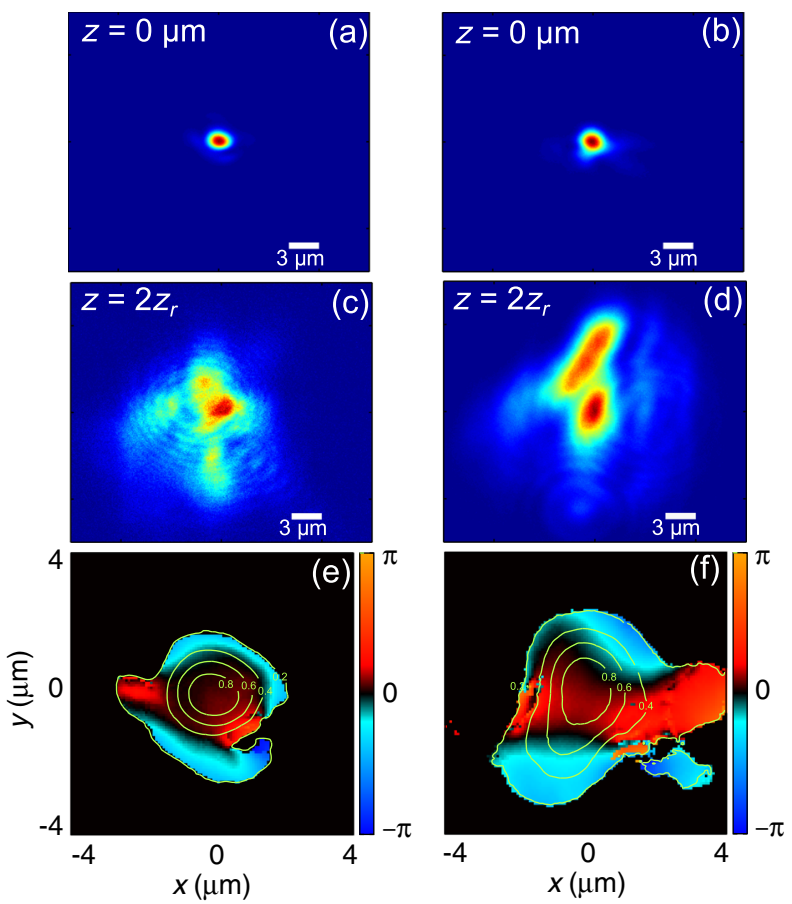

FIG. 3. Laser focal spots and wave fronts. (a),(b) Laser focal spots for two different runs. (c),(d) Corresponding midfield laser transverse distribution at $z=2 z_{R}$. (e),(f) Corresponding reconstructed laser phase at the focal plane.

\section{PIC SIMULATIONS}

To understand how the wave front distortions actually affect the electron acceleration, we perform PIC simulations including realistic laser wave fronts. We use the code CALDER-CIRC [27], a fully electromagnetic 3D code based on cylindrical coordinates $(r, z)$ and Fourier decomposition in the poloidal direction. The simulations are performed using a mesh with $\Delta z=0.3 k_{0}^{-1}$ and $\Delta r=1.5 k_{0}^{-1}$ (where $k_{0}$ is the laser wave vector) and the seven first Fourier modes. The neutral gas density profile is taken from the experimental data. The simulations start with pure neutral nitrogen, which is ionized via tunnel ionization. The number of macroparticles per cell before ionization is 2000 , which corresponds to $2000 \times 5=10000$ macroelectrons per cell in the region of full ionization of the $L$ shell of nitrogen. We perform simulations on two test cases. In the first one, the laser focal spot is taken from the experimental data and the wave front is reconstructed using the Gerchberg-Saxton algorithm. In the second one, the same experimental focal spot is used, but the wave front is assumed to be perfectly flat. The wave front quality has a dramatic effect on the midfield intensity distribution (see also Supplemental Material for more details [16]).

PIC simulations show that electrons are injected in the wakefield generated in the downward density gradient. This can be seen in Fig. 4, which shows the map of the electron density in the plane $y=0$ and the electron distribution in phase space $\left(z, v_{z}\right)$ for two times,
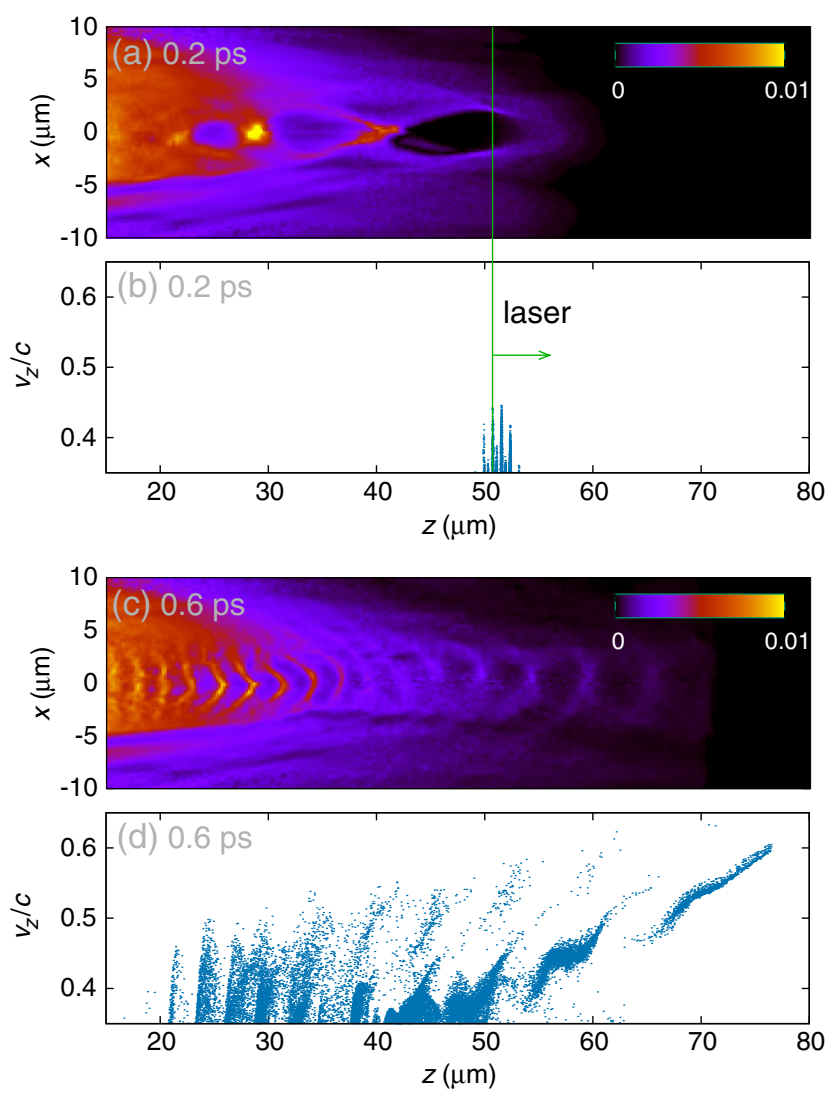

FIG. 4. Results of 3D PIC simulations with reconstructed laser wave front. Electron density $n_{e} / n_{c}$ in the downward density ramp at the exit of the gas jet at (a) 0.2 and (c) 0.6 ps. (b), (d) Corresponding electron longitudinal phase space showing electron trapping and acceleration in the wakefield.

corresponding to the simulation with the reconstructed wave front. The figure displays only the region at the exit of the gas jet ( $z=0$ is the center of the jet). The laser pulse, whose focal plane is at $z \sim 43 \mu \mathrm{m}$, is close to resonance in this region, and therefore it excites a large amplitude wakefield [Fig. 4(a)]. In addition, comparing the density maps at 0.2 and 0.6 ps, Figs. 4(a) and 4(c), clearly shows that the wavelength of the plasma wave decreases with time. This reduction of the plasma wavelength (i.e., increase of the plasma wave vector) is a known feature of plasma wave excitation in a density gradient. It leads to the decrease of the phase velocity $v_{p}$, which eventually leads to the trapping and injection of electrons in the wakefield [19,21] (see also the Appendix for more details).

The analysis of the simulations confirms that electrons are injected when the phase velocity is sufficiently low. At early times, $v_{p} \simeq c$, and there are no longitudinally accelerated electrons in the wakefield buckets closest to the laser pulse, as can be seen in the phase space for $t=$ 0.2 ps [Fig. 4(b)]. The only electrons with large longitudinal velocity are those oscillating in the high-frequency laser field. The simulation shows that trapping occurs later, when the phase velocity reaches $v_{p} / c \simeq 0.45$; see Fig. 4 (d). 
This low phase velocity explains why electrons are accelerated in the 100-keV energy range. Note also that trapping occurs after the laser pulse has passed; i.e., the electron bunch sits $\simeq 70 \mu \mathrm{m}$ behind the laser pulse and never interacts with the laser field. Electrons are trapped in several buckets of the wakefield, as shown in the phase space snapshot at 0.6 ps [Fig. 4(d)]. Finally, the energy distribution at the exit of the plasma agrees very well with the experiment [see Fig. 2(f)], which confirms the trapping and acceleration mechanism.

The trends described above are also found in the simulations using a flat wave front. However, differences are dramatic when inspecting the shape of the electron transverse distribution obtained in each case. We now focus on the impact of the transverse wakefield $E_{\perp}=\sqrt{E_{x}^{2}+E_{y}^{2}}$ on the electron distribution. When the experimental laser spot and the reconstructed wave front are used [Fig. 5(a)], the cross sections of the transverse wakefield $E_{\perp}$ are highly asymmetric and the electron distribution is complex, with hot spots and regions without electrons. This computed electron distribution is similar to the experimental ones [Figs. 2(c) and 2(d)]. For the case with a flat wave front, Fig. 5(b), the cross section of the transverse wakefield is more rotationally symmetric, and as a result, the electron bunch is much more collimated. This flat phase case resembles the measured electron beam image shown in Fig. 2(e), indicating that on that particular experimental run the wave front is less distorted.

The total charge $(\sim 40 \mathrm{fC}$, close to the experimental result) is similar when a flat or distorted wave front is used, but the crucial difference is that a flat wave front yields an electron beam contained within a small solid angle $\lesssim 0.05 \mathrm{sr}$, compared to $\sim 0.6 \mathrm{sr}$ for the experimental wave front. Note that in both cases the intensity distributions at the focal plane are the same, only the wave front differs.

(a)
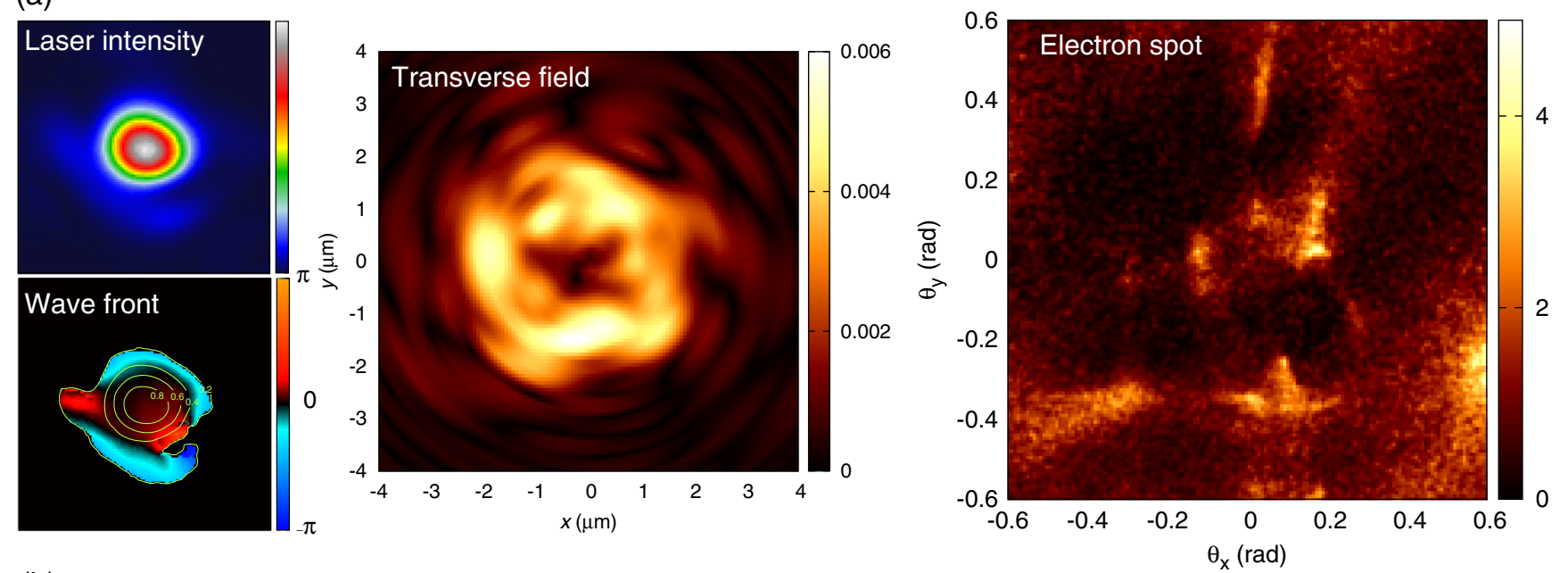

(b)
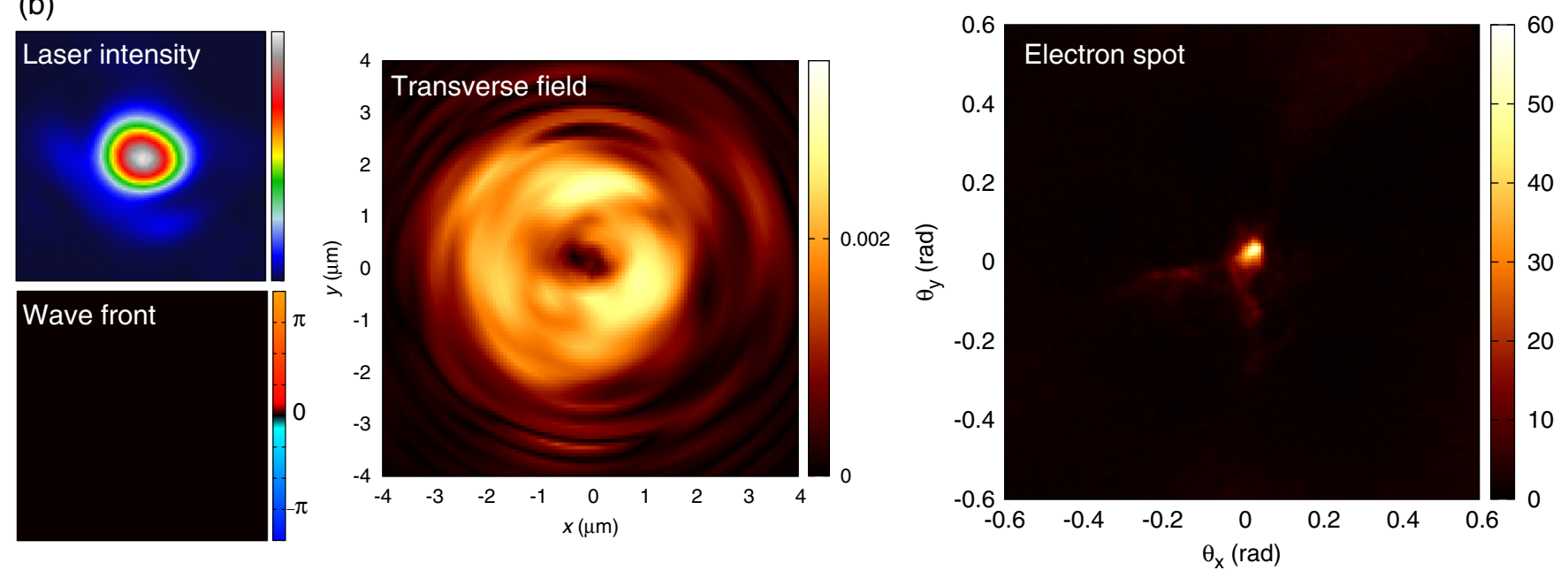

FIG. 5. Simulations results for a laser pulse with both focal spot and wave front reconstructed from experimental data (a) and with the same experimental focal spot but with a flat wave front (b) Left column: input intensity distribution and wave front at the focal plane. Central column: cross-sections of the transverse field $E_{\perp} / E_{0}\left(E_{0}=m_{e} c \omega_{p} / e\right.$ is the cold wave breaking field $)$ at 0.6 ps and for $z=28 \mu \mathrm{m}$. Right column: electron spot far from the gas jet. 
These results indicate that the transverse wakefield inhomogeneities act as focusing or defocusing electron optics, resulting in the observed complex patterns. A good electron beam quality can be obtained when the transverse inhomogeneities are reduced over the acceleration length, i.e., a few Rayleigh lengths.

We find that the transverse inhomogeneities originate mostly from the distorted laser wave front, and not from ionization-induced nonlinear propagation effects. Indeed, both our experimental measurements (see Supplemental Material [16]) and simulations show that the laser pulse and the wakefield do not undergo any significant distortion associated with ionization. However, it is quite clear that the phase distortion at the focal plane greatly affects the midfield intensity profile of the laser beam (see Fig. 3), and therefore the shape of the wakefield. The structured electron distribution and the structure of the wakefield thus come as a result of the midfield inhomogeneities induced by the distorted wave front. This interpretation is also supported by the fact that our simulations with a flat wave front and the same focal spot yield a much more collimated and symmetric electron beam, even though ionization is present. Another effect that we can rule out is beam-plasma filamentation [28], as the structures in the electron beam disappear when using the flat laser wave front. Thus, the laser wave front has a direct effect on the electron beam distribution. This suggests that tailoring the wave front permits the control of the focusing properties of the transverse wakefield. This also explains the results of Refs. [8,29], where a deformable mirror was used in order to modify the wave front and optimize the electron beam quality.

In conclusion, we observe and explain the complex electron distributions in a high-repetition rate laser-plasma accelerator. PIC simulations indicate that these complex distributions are related to distortions of the laser wave front, which cause inhomogeneities in the laser distribution and the transverse wakefield. Our work emphasizes the importance of using realistic laser distributions in simulations in order to understand and reproduce experimental results quantitatively. In addition, while our experiment operates in an extreme regime where the interaction length is large compared to the Rayleigh length, it demonstrates the importance of controlling the laser wave front over several Rayleigh lengths in laser-plasma interaction experiments. These results might also be relevant to the case of higher energy laser-plasma accelerators, even when selffocusing governs the laser propagation: recent work mentions the importance of the laser intensity in the midfield $[30,31]$. Finally, our results suggest that the laser wave front is a knob for actively tuning the electron beam distribution, which can be achieved with a deformable mirror, for instance $[8,29]$. This fact, along with the stability and the $\mathrm{kHz}$ repetition rate make this electron source attractive for applications such as time-resolved electron diffraction $[10,12]$.

\section{ACKNOWLEDGMENTS}

This work was funded by the European Research Council under Contract No. 306708, ERC Starting Grant FEMTOELEC, and the Agence Nationale pour la Recherche under Contract No. ANR-11-EQPX-005ATTOLAB

\section{APPENDIX: WAKEFIELD PHASE VELOCITY IN A DENSITY GRADIENT}

Our experimental data and PIC simulations indicate that electrons are injected in the wakefield in the downward density ramp, at the exit of the gas jet. The trapping and the acceleration of electrons in a density down ramp were studied theoretically in Ref. [19], where it was shown that the local wave number of the wakefield increases over time:

$$
k(z, t)=k_{p}(z)+(z-c t) \frac{d k_{p}}{d z}
$$

where $z$ is the longitudinal coordinate, $c$ is the speed of light, $k_{p}(z)=\omega_{p}(z) / c$, and $\omega_{p}(z)=\sqrt{n_{e}(z) e^{2} / \epsilon_{0} m_{e}}$ is the $z$-dependent plasma frequency in the density gradient. It is important to note that, contrary to the wave vector $k(z, t)$, $\omega_{p}(z)$ does not evolve with time. As a result of this timevarying wave vector, the phase velocity $v_{p}(z, t)=$ $\omega_{p} / k(z, t)$ of the wakefield generated by a laser focused in a density down ramp decreases with time as

$$
v_{p}(z, t)=c \frac{1}{1+(z-c t) \frac{1}{k_{p}} \frac{d k_{p}}{d z}} .
$$

Indeed, for a downward density ramp, $d k_{p} / d z<0$, and behind the laser pulse $z-c t<0$, so that the denominator in this equation is larger than 1 . Consequently, as the wave vector increases with time, the phase velocity decreases. Injection occurs behind the laser pulse when the wakefield becomes slow enough to trap plasma background electrons.

[1] E. Esarey, P. Sprangle, J. Krall, and A. Ting, Overview of Plasma-Based Accelerator Concepts, IEEE Trans. Plasma Sci. 24, 252 (1996).

[2] E. Esarey, C. B. Schroeder, and W. P. Leemans, Physics of Laser-Driven Plasma-Based Electron Accelerators, Rev. Mod. Phys. 81, 1229 (2009).

[3] J. Faure, C. Rechatin, A. Norlin, A. Lifschitz, Y. Glinec, and V. Malka, Controlled Injection and Acceleration of Electrons in Plasma Wakefields by Colliding Laser Pulses., Nature (London) 444, 737 (2006).

[4] W. P. Leemans, B. Nagler, A. J. Gonsalves, Cs. Tòth, K. Nakamura, C. G. R. Geddes, E. Esarey, C. B. Schroeder, and S. M. Hooker, GeV Electron Beams from a CentimetreScale Accelerator, Nat. Phys. 2, 696 (2006). 
[5] O. Lundh, J. Lim, C. Rechatin, L. Ammoura, A. Ben-Ismail, X. Davoine, G. Gallot, J.-P. Goddet, E. Lefebvre, V. Malka, and J. Faure, Few Femtosecond, Few Kiloampere Electron Bunch Produced by a Laser-Plasma Accelerator, Nat. Phys. 7, 219 (2011).

[6] G. Sun, E. Ott, Y. C. Lee, and P. Guzdar, Self-Focusing of Short Intense Pulses in Plasmas, Phys. Fluids 30, 526 (1987).

[7] A. B. Borisov, A. V. Borovskiy, O. B. Shiryaev, V. V. Korobkin, A. M. Prokhorov, J. C. Solem, T. S. Luk, K. Boyer, and C. K. Rhodes, Relativistic and Charge Displacement Self-Channeling of Intense Ultrashort Laser Pulses in Plasmas, Phys. Rev. A 45, 5830 (1992).

[8] Z.-H. He, B. Hou, J. H. Easter, J. Faure, K. Krushelnick, J. A. Nees, and A. G. R. Thomas, High Repetition-Rate Wakefield Electron Source Generated by Few-Millijoule, 30 Femtosecond Laser Pulses on a Density Downramp, New J. Phys. 15, 053016 (2013).

[9] B. Beaurepaire, A. Lifschitz, and J. Faure, Electron Acceleration in Sub-Relativistic Wakefields Driven by Few-Cycle Laser Pulses, New J. Phys. 16, 023023 (2014).

[10] G. Sciaini and R. J. D. Miller, Femtosecond Electron Diffraction: Heralding the Era of Atomically Resolved Dynamics, Rep. Prog. Phys. 74, 096101 (2011).

[11] P. Musumeci, J. T. Moody, C. M. Scoby, M. S. Gutierrez, and M. Westfall, Laser-Induced Melting of a Single Crystal Gold Sample by Time-Resolved Ultrafast Relativistic Electron Diffraction, Appl. Phys. Lett. 97, 063502 (2010).

[12] Z.-H. He, A. G. R. Thomas, B. Beaurepaire, J. A. Nees, B. Hou, V. Malka, K. Krushelnick, and J. Faure, Electron Diffraction Using Ultrafast Electron Bunches from a LaserWakefield Accelerator at $\mathrm{kHz}$ Repetition Rate, Appl. Phys. Lett. 102, 064104 (2013).

[13] A. Jullien, A. Ricci, F. Böhle, J.-P. Rousseau, S. Grabielle, N. Forget, H. Jacqmin, B. Mercier, and R. Lopez-Martens, Carrier-Envelope-Phase Stable, High-Contrast, Double Chirped-Pulse-Amplification Laser System, Opt. Lett. 39, 3774 (2014).

[14] R. Rankin, C. E. Capjack, N. H. Burnett, and P. B. Corkum, Refraction Effects Associated with Multi-Photon Ionisation and Ultra-Short Pulse Laser Propagation in Plasma Waveguides, Opt. Lett. 16, 835 (1991).

[15] P. Chessa, E. De Wispelaere, F. Dorchies, V. Malka, J.-R. Marquès, G. Hamoniaux, P. Mora, and F. Amiranoff, Temporal and Angular Resolution of the Ionization-Induced Refraction of a Short Laser Pulse in a Helium Gas, Phys. Rev. Lett. 82, 552 (1999).

[16] See Supplemental Material at http://link.aps.org/ supplemental/10.1103/PhysRevX.5.031012 for informations concerning the laser beam propagation in the plasma and the reconstruction of the laser wave front.

[17] D. Teychenné, G. Bonnaud, and J.-L. Bobin, WaveBreaking Limit to the Wake-Field Effect in an Underdense Plasma, Phys. Rev. E 48, R3248 (1993).

[18] S. Bulanov, N. Naumova, F. Pegoraro, and J. Sakai, Particle Injection into the Wave Acceleration Phase due to Nonlinear Wake Wave Breaking, Phys. Rev. E 58, R5257 (1998).
[19] A. V. Brantov, T. Zh. Esirkepov, M. Kando, H. Kotaki, V. Yu. Bychenkov, and S. V. Bulanov, Controlled Electron Injection into the Wake Wave Using Plasma Density Inhomogeneity, Phys. Plasmas 15, 073111 (2008).

[20] C. G. R. Geddes, K. Nakamura, G. R. Plateau, Cs. Tóth, E. Cormier-Michel, E. Esarey, C. B. Schroeder, J. R. Cary, and W. P. Leemans, Plasma-Density-Gradient Injection of Low Absolute-Momentum-Spread Electron Bunches, Phys. Rev. Lett. 100, 215004 (2008).

[21] J. Faure, C. Rechatin, O. Lundh, L. Ammoura, and V. Malka, Injection and Acceleration of Quasimonoenergetic Relativistic Electron Beams Using Density Gradients at the Edges of a Plasma channel, Phys. Plasmas 17, 083107 (2010).

[22] K. Schmid, A. Buck, C. M. S. Sears, J. M. Mikhailova, R. Tautz, D. Herrmann, M. Geissler, F. Krausz, and L. Veisz, Density-Transition Based Electron Injector for Laser Driven Wakefield Accelerators, Phys. Rev. ST Accel. Beams 13, 091301 (2010).

[23] C. McGuffey, A. G. R. Thomas, W. Schumaker, T. Matsuoka, V. Chvykov, F. J. Dollar, G. Kalintchenko, V. Yanovsky, A. Maksimchuk, K. Krushelnick, V. Yu. Bychenkov, I. V. Glazyrin, and A. V. Karpeev, Ionization Induced Trapping in a Laser Wakefield Accelerator, Phys. Rev. Lett. 104, 025004 (2010).

[24] A. Pak, K. A. Marsh, S. F. Martins, W. Lu, W. B. Mori, and C. Joshi, Injection and Trapping of Tunnel-Ionized Electrons into Laser-Produced Wakes, Phys. Rev. Lett. 104, 025003 (2010).

[25] These statistics were obtained by analyzing a sequence of data where each point was the result of an average over 500-1000 shots.

[26] R. W. Gerchberg and W. O. Saxton, A Practical Algorithm for the Determination of the Phase from Image and Diffraction Plane Pictures, Optik (Stuttgart) 35, 237 (1972).

[27] A. F. Lifschitz, X. Davoine, E. Lefebvre, J. Faure, C. Rechatin, and V. Malka, Particle-in-Cell Modelling of Laser-Plasma Interaction Using Fourier Decomposition, J. Comput. Phys. 228, 1803 (2009).

[28] M.-C. Firpo, A. F. Lifschitz, E. Lefebvre, and C. Deutsch, Early Out-of-Equilibrium Beam-Plasma Evolution, Phys. Rev. Lett. 96, 115004 (2006).

[29] Z.-H. He, B. Hou, V. Lebailly, J. A. Nees, K. Krushelnick, and A. G. R. Thomas, Coherent Control of Plasma Dynamics, Nat. Commun. 6, 7156 (2015).

[30] X. Wang et al., Quasi-Monoenergetic Laser-Plasma Acceleration of Electrons to $2 \mathrm{GeV}$, Nat. Commun. 4, 1988 (2013).

[31] W. P. Leemans, A. J. Gonsalves, H.-S. Mao, K. Nakamura, C. Benedetti, C. B. Schroeder, Cs. Tóth, J. Daniels, D. E. Mittelberger, S. S. Bulanov, J.-L. Vay, C. G. R. Geddes, and E. Esarey, Multi-GeV Electron Beams from Capillary-Discharge-Guided Subpetawatt Laser Pulses in the Self-Trapping Regime, Phys. Rev. Lett. 113, 245002 (2014). 